\title{
The economic impact of inflammatory bowel disease in Canada
}

\author{
Robert Hilsden MD PhD FRCPC
}

\begin{abstract}
ARTICLE
Longobardi T, Jacobs P, Wu L, Bernstein CN. Work losses related to inflammatory bowel disease in Canada: Results from a National Population Health Survey. Am J Gastroenterol 2003;98:844-9.
\end{abstract}

\begin{abstract}
ARTICLE SUMMARY
Longobardi and colleagues examined the effect of inflammatory bowel disease (IBD) on employment, using data from 10,891 respondents aged 20 to 64 years from the 1998 cycle of the Canadian National Population Health Survey (NPHS) (1). This sample included 187 (1.7\%) subjects who self-reported IBD or a similar bowel disorder. A significantly greater proportion of IBD than non-IBD respondents reported that they were not in the labour force (28.9\% versus $18.5 \%$ ). Even after adjusting for other factors (age group, level of pain, etc), subjects with IBD had a $2.9 \%$ higher nonparticipation rate $(21.4 \%)$. For example, among people not hospitalized within the past year and with no limitation of activities due to pain, IBD subjects were 1.2 times more likely to be unemployed than those without IBD. Subjects who reported high levels of pain had a very high probability of being out of the labour force. Based on Canadian annual compensation data for all employed persons in Canada, and age- and sex-specific prevalence, and incidence rates for IBD, the authors estimated that there are 119,980 IBD patients between the ages of 20 and 64 years in Canada and that this group includes 3479 people who are not in the labour force. This translates into lost wages of $\$ 104.2$ million, or $\$ 868$ per IBD patient
\end{abstract}

\section{COMMENTARY}

There is a serious lack of data on the economic and social impacts of IBD. This information is important for understanding the burden of suffering experienced by IBD patients, and for evaluating the cost effectiveness of therapies. Emerging therapies, especially for Crohn's disease, are expensive and usually not affordable 'out-ofpocket' by most disease sufferers. Therefore, their costs are shifted to health regions, provincial ministries of health and other third party payers. Because resources are limited, payers must decide whether a given therapy should be provided. Often, an important factor in making these decisions depends on the therapy's cost and the potential to deliver results; its 'bang for the buck', which is often estimated from cost effectiveness models. Direct health costs (physician fees, drugs and hospitalization costs) are relatively easy to measure. In contrast, data on indirect costs (patient's outof-pocket expenses, lost wages, etc) are not readily available. Therefore, economic evaluations of IBD treatments, such as infliximab, are limited in terms of the quality and completeness of information. Cost effectiveness studies of infliximab for rheumatoid arthritis have routinely included indirect costs, whereas similar studies in Crohn's disease have not (2).

Longobardi and colleagues have conducted a methodologically rigorous study that provides a wealth of important Canadian data on the economic impact of bowel disease. The limitations of the study are due to the limitations of the NPHS.

One goal of the NPHS, conducted by Statistics Canada, was to collect data on the economic, social, demographic, occupational and environmental correlates of health. The survey elicited whether respondents suffered from a list of several chronic medical conditions. In the preface to the question, it was made clear that only "long-term conditions" that had lasted or were expected to last six months or more, and that were diagnosed by a health professional, should be included.

Longobardi classified respondents as having or not having IBD or a similar disorder, based on the question, "Do you have a bowel disorder such as Crohn's disease or colitis?" I believe that identifying those who answered yes to this question as having "IBD" or even "IBD or a similar disorder" is overly optimistic. Compare this question to the much more specific question used in the US National 
Health Interview Survey (NHIS): "Have you been told by a doctor or other health professional that you had Crohn's disease or ulcerative colitis?" (3). A respondent might interpret the NPHS question as just giving examples of bowel diseases, whereas another respondent might interpret the question as being restricted to Crohn's disease or colitis. Of course, the term 'colitis' is itself vague. Clearly, there is a great potential for the misclassification of people with other bowel disorders, most importantly irritable bowel syndrome, as having IBD. The authors address this in their discussion. They note that the prevalence of IBD from the NPHS is more than twice than expected, based on population-based data from Manitoba. Therefore, the question is not "Is there significant misclassification of patients?", but rather "Has this significantly biased the results?" The authors argue that their data provide a "minimum estimate of the influence of IBD on work loss." This is probably a reasonable assumption, but deserves to be tested in further studies. This same group of authors using the US NHIS found a much higher rate of labour force nonparticipation attributable to IBD (3). A more conservative interpretation of the NPHS data would be that it provides employment data on those with a variety of bowel conditions, including IBD. This is a matter of semantics, I realize, but I believe it is a more realistic interpretation of the data, given the limitations of the NHPS.

The potential impact of IBD misclassification is mitigated by the authors' ability to stratify respondents by the degree of pain and hospitalization within the past 12 months. Novel therapies for IBD would predominantly be used in those with more severe disease. Therefore, economic analyses of these novel therapies need accurate data about the medical and societal costs of disease in this severely affected group. We would be concerned about using data in an economic analysis that might be significantly contaminated by those with relatively mild cases of irritable bowel syndrome. This is of much less concern when we are able to use data from more severely ill subjects, as measured by impairment of normal activities or a history of hospitalization.

Future work should seek to accurately define the effect of IBD not only on labour force nonparticipation, but also on other important economic parameters, such as underemployment, unpaid employment (housework) and educational attainment, and noneconomic outcomes such as quality of life and psychological well-being. Large national surveys provide one means of achieving these goals.

The NPHS provides a wealth of data. It has been underused by those interested in gastrointestinal diseases. A Medline search reveals 173 articles using NPHS data published since 1996. Only three of these involved gastrointestinal disorders (Longobardi is the author of two these). The cross-sectional component of the NPHS has now been discontinued and replaced by the Canadian Community Health Survey (CCHS). The CCHS includes a much larger sample size (more than 130,000 participants), which allows comparisons to be made at subprovincial levels of geography. However, the questions about chronic conditions remain vague and gastrointestinal diseases are still not adequately addressed (4). Use of mammography, pap smear tests, and prostate-specific antigen tests were included in the common content of the first cycle of the CCHS, but colorectal cancer screening was not included. Cycle two data will be released in summer 2004. An optional colorectal cancer screening module was available for cycle two, but it remains to be seen what proportion of health regions decided to include it. Data collection for cycle three begins in January 2005. In the fall of 2003, Statistics Canada sought input from stakeholders, including researchers, on the content of cycle three. I hope that some members of the Canadian gastrointestinal research community participated in this process. This participation must increase if the CCHS is to meet its potential to provide relevant and accurate information on the determinants and impacts of gastrointestinal diseases in Canada.

\section{REFERENCES}

1. Longobardi T, Jacobs P, Wu L, Bernstein CN. Work losses related to inflammatory bowel disease in Canada: Results from a National Population Health Survey. Am J Gastroenterol 2003;98:844-9.

2. Marshall JK, Blackhouse G, Goeree R, et al. Infliximab for the treatment of Crohn's disease: A systematic review and cost-utility analysis. Technology report No. 24, 2002. Ottawa: Canadian Coordinating Office for Health Technology Assessment.

3. Longobardi T, Jacobs P, Bernstein CN. Work losses related to inflammatory bowel disease in the United States: Results from the National Health Interview Survey. Am J Gastroenterol 2003;98:1064-72.

4. Statistics Canada. Canadian Community Health Survey Cycle 2.1 Common content modules.

$<$ http://www.statcan.ca/english/concepts/health/cycle2_1/common. htm $>$ (Version current at February 27, 2004).

\section{The authors respond:}

The importance of using population-based data, as found in Canada's National Population Health Survey (NPHS) and the more recent Canadian Community Health Survey (CCHS), is emphasized by Dr Hilsden in his insightful commentary. Upon completing our study of the indirect cost of inflammatory bowel disease (IBD) using the NPHS (1), we undertook a three-part empirical analysis of the 1999 US National Health Interview Survey (NHIS) (2). We pursued a comprehensive analysis of the US NHIS data instead of its Canadian counterpart, the NPHS, because of the superiority of the self-identifying question and its questions about two other parameters: (a) symptomatic IBD in the past year, and (b) the age of the respondent at the onset of IBD. Using these, together with other demographic variables, we present three methodologies to study the economics of IBD by quantifying indirect cost (3), excess utilization (4) and lifetime excess utilization (5).

As explained by Dr Hilsden, the question used to identify IBD respondents in the NPHS was too broad, likely capturing individuals with bowel disorders 'similar to' IBD. Hence, while our initial study using the NPHS gives an estimate of indirect costs of IBD and similar diseases in Canada, it undoubtedly does include some cases of irritable 
bowel syndrome (IBS) or other non-IBD disorders. Nonetheless, it does quantify indirect costs for a group of patients with gastrointestinal symptoms, many of whom likely have IBD. On the basis of our population-based data in Manitoba (6), we suspect that the NPHS overestimates the true prevalence of IBD. However, the NHIS question "Have you EVER been told by a doctor or other health professional that you had Crohn's disease or ulcerative colitis?" identifies adult IBD cases by naming specifically its two predominant forms. The validity of the NHIS questions is reflected by the fact that the survey yields an estimate of the prevalence of IBD that approximates that reported in the US adult population (6)

In spite of their shortcomings, the key advantage to using these types of surveys is that they provide national population-based data. Unfortunately, the 2002 NHIS lumps IBD with IBS and severe constipation requiring medication. As the US NHIS appears to be moving away from differentiating IBD from other bowel conditions, the Canadian national survey (CCHS) is considering moving towards a more targeted question to capture information on IBD. Since our work on the NPHS, the NPHS methodologists have been encouraged by our group and others to provide a more targeted question. Methodologists have argued that they are interested in a class of digestive conditions that are 'similar to' IBD. For the purpose of cost analysis, however, we believe that the per capita cost for IBD would be greater than for IBS, since some cases of Crohn's disease, in particular, are expensive to treat.
There is clearly an argument to distinguish IBD from IBS for the purpose of cost analysis. We are grateful that Dr Hilsden is adding his voice for refinement of the work in this area, including a better question in Canadian national surveys. We hope that this will help prompt the development of better economic data.

Teresa Longobardi PhD

Charles $N$ Bernstein MD

University of Manitoba Inflammatory Bowel Disease Clinical and Research Centre and Department of Internal Medicine, Winnipeg, Manitoba

\section{REFERENCES}

1. Longobardi T, Jacobs P, Wu L, Bernstein CN. Work losses related to inflammatory bowel disease in Canada: Results from a National Population Health Survey. Am J Gastroenterol 2003;98:844-9.

2. Centers for Disease Control and Prevention. 1999 National Health Interview Survey (NHIS) Public Use Data Release.

<ftp://ftp.cdc.gov/pub/Health_Statistics/NCHS/Dataset_Documentat ion/NHIS/1999/srvydesc.pdf> (Version current at March 5, 2004).

3. Longobardi T, Jacobs P, Bernstein CN. Work losses related to inflammatory bowel disease in the United States: Results from the National Health Interview Survey. Am J Gastroenterol 2003;98:1064-72.

4. Longobardi T, Jacobs P, Bernstein CN. Utilization of health-care resources by individuals with inflammatory bowel disease in the US: A profile of time since diagnosis. Am J Gastroenterol 2004. (In press)

5. Longobardi T, Jacobs P, Bernstein CN. Utilization of health-care resources by individuals with inflammatory bowel disease in the US: an application of the two-part model. Submitted to Journal of Health Economics.

6. Bernstein CN, Blanchard JF, Rawsthorne P, Wajda A. Epidemiology of Crohn's disease and ulcerative colitis in a central Canadian province: A population-based study. Am J Epidemiol 1999;149:916-24. 


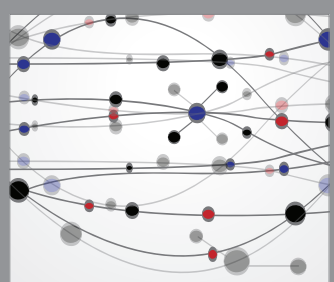

The Scientific World Journal
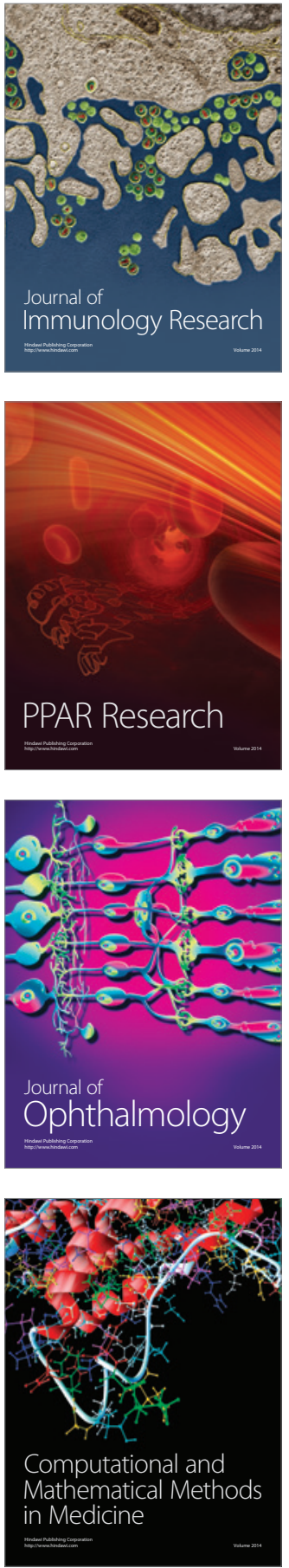

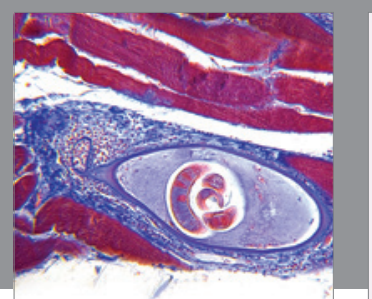

Gastroenterology Research and Practice

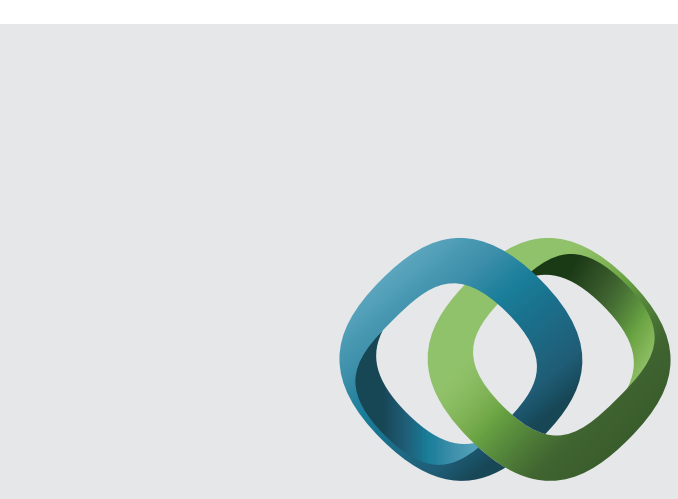

\section{Hindawi}

Submit your manuscripts at

http://www.hindawi.com
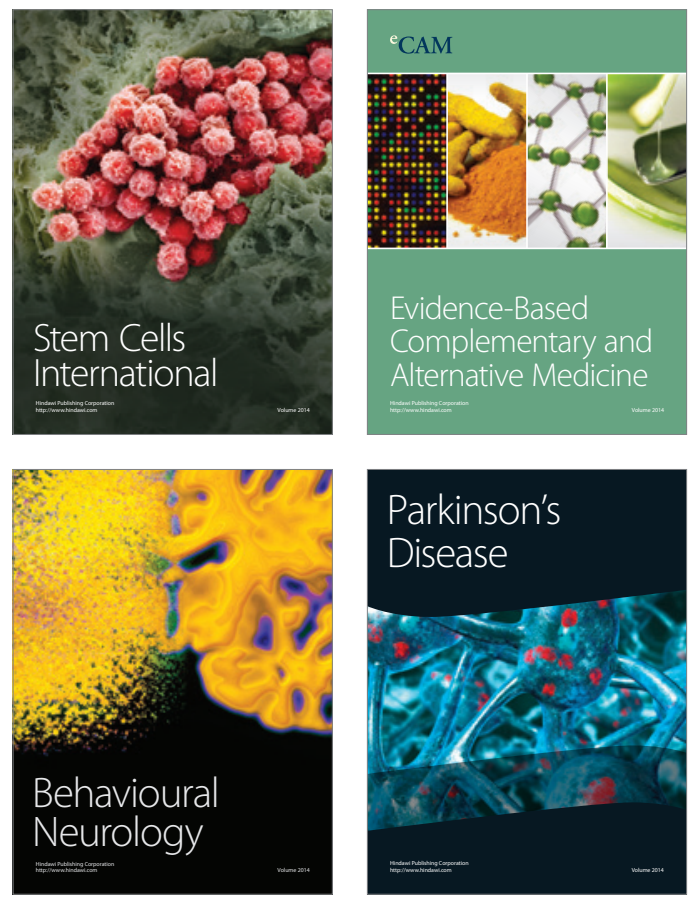
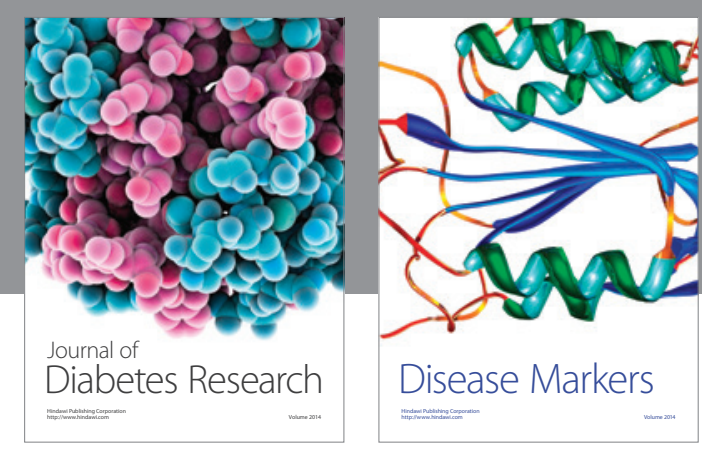

Disease Markers
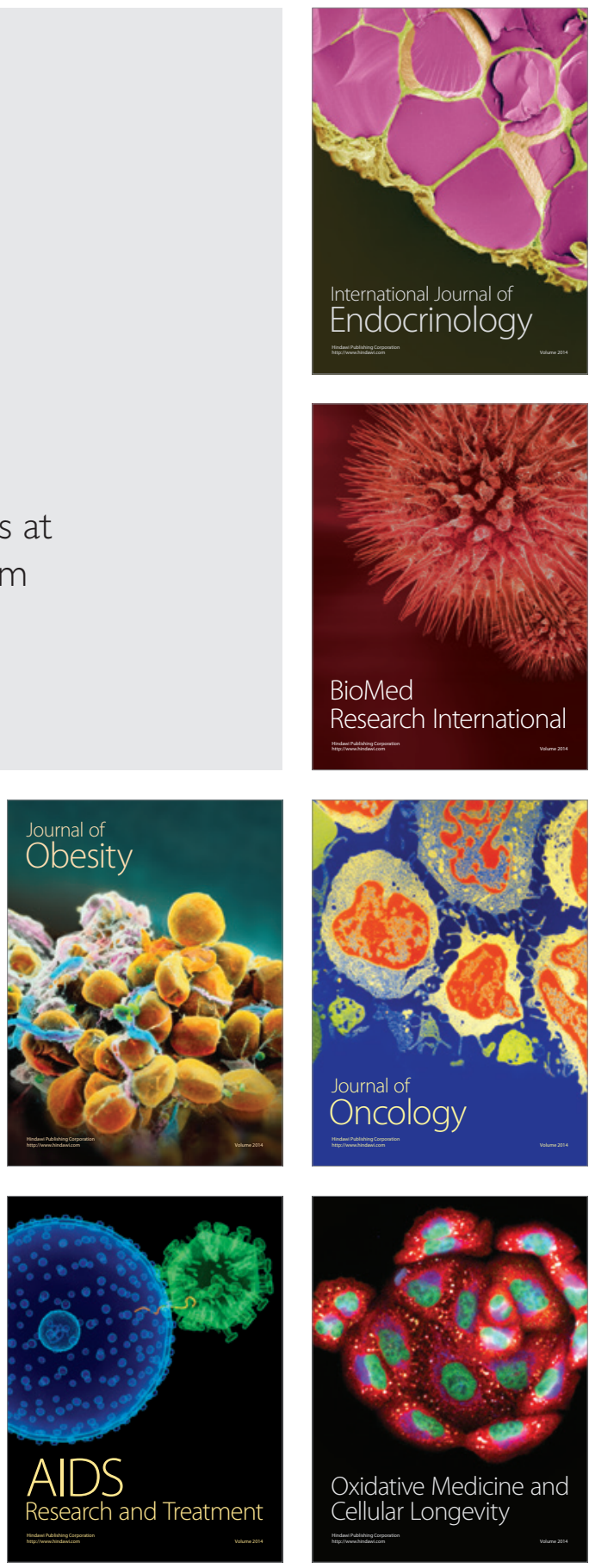